Parkinson's disease can employ a predictive motor strategy. J Neurol Neurosurg Psychiatry 1984;47:1299-306.

${ }^{2}$ Flowers KA. Lack of prediction in the motor behavior of Parkinsonism. Brain 1978; 101:35-52.

${ }^{3}$ Stern Y, Mayeux R, Rosen J, Ilson J. Perceptual motor dysfunction in Parkinson's disease: A deficit in sequential and predictive voluntary movement. J Neurol Neurosurg Psychiatry 1983;46:145-51.

${ }^{4}$ Stern Y, Mayeux R, Rosen J. Contribution of perceptual motor dysfunction to construction and tracing disturbances in Parkinson's disease. J Neurol Neurosurg Psychiatry 1984;47:983-94.

${ }^{5}$ Bloxham CA, Mindel TA, Frith CD. Initiation and execution of predictable and unpredictable movements in Parkinson's disease. Brain 1984;107:371-84.

\section{Day et al reply}

We thank Dr Stern for his comments and suggestions, but still believe that our conclusions hold. As stated in the final paragraph of the discussion to our paper, we think that the data show that patients with Parkinson's disease can and do adopt a predictive motor strategy when tracking a known target movement, but that they do so with less accuracy than normal subjects.

The issue of short-term prediction of unknown targets, which leads to tracking lags shorter than visual reaction times in both normals and Parkinsonians, is not the crucial point. It is the ability of Parkinsonians to reduce tracking lag closer to zero when the target movement is known that defines the use of a predictive motor strategy, and 11 of the 12 patients studied were able to do this. If, as suggested by Dr Stern, the patients and controls are compared by measuring the reduction in lag for each subject, then a $t$ test does reveal a difference between the groups at the $5 \%$ level of significance $(t=2 \cdot 29, \mathrm{p}=0.33$ for a twotailed test). ANOVA gives essentially the same result with a significant main effect of conditions $(F=46.88, p<0.001)$ but not of groups $(F=0.05, p>0.05)$ with a significant interaction between groups and conditions $(F=5 \cdot 24, p=0.034)$. However, it should be pointed out that strictly speaking a $t$ test is not applicable to these data. The difference in variability of the two groups is large (variance ratio $=6.24$ ) and the control data is skewed. The nonparametric Mann-Whitney test is more suitable but fails to reveal a difference between the groups $(U=23, p>0.05)$. Therefore, we believe that the present data are inconclusive on this point, and that this issue can be resolved only by further experimentation.
Our data show that even though a predictive strategy was used, the tracking error of the patient group did not improve as much as the control group. There are two possible ways of explaining this. Either the patients tracking lag did not improve as much as controls', or, lag was reduced normally but the movements were more inaccurate. In contrast to Dr Stern, we favour the latter explanation for the following reasons: (1) The mean tracking lag of the patients was not statistically different from that of the controls in both the unpredictable and predictable conditions. (2) There was a correlation between the improvement in error and reduction in lag for the control subjects $(r=0.83, p<0.05)$, but not for the patient group $(r=0.03, p>0.05)$.

In conclusion, Dr Stern and we agree that patients with Parkinson's disease can adopt predictive movement, but less successfully than normal subjects.

\section{Triphasic waves of metabolic encephalopathy versus spike-wave stupor}

Sir: We disagree with the discussion that Yamamoto and Hosokawa give in their recent case report "Triphasic spike-wave stupor in portal-systemic encephalopathy."1 They seem to confuse the EEG findings of triphasic waves seen in metabolic disturbances and spike-wave discharges seen in epileptic spike-wave stupor (also called absence status or petit mal status). This distinction has diagnostic and therapeutic importance.

Their patient was a 59-year-old woman presenting with episodic obtundation and a flapping tremor. Portal-systemic encephalopathy was diagnosed from laboratory and radiologic evidence of liver cirrhosis (including hyperammonaemia) and treated with lactulose and kanamycin. They concluded from EEG findings of "continuous bilaterally synchronous atypical spike-wave discharges of an epileptic nature" that the patient had spike-wave stupor secondary to portal-systemic encephalopathy.

A more accurate interpretation of the EEG (shown in the figure accompanying the case report) would be triphasic waves suggestive of a metabolic disturbance. Triphasic waves are medium to high amplitude, diffuse, frontally predominant, broad complexes consisting of a large positive (downward) sharp wave preceded and followed by small negative (upward) components. ${ }^{2}$ These complexes last 0.15 to 0.25 seconds and recur singly or in serial trains about every 0.5 to 2 seconds. They are often super- imposed on a background of asynchronow and bisynchronous slow waves. There is usially a tendency for fronto-occipital delay. Although rarely seen in degenerative processes or after anoxic episodes and hegis trauma, triphasic waves usually indicate toxic-metabolic encephalopathy, most coifmonly hepatic or renal. They are not efileptic phenomena. ${ }^{3}$ There appears to be limited correlation between blood ammoxi levels and the triphasic wave pattern. ${ }^{4}$ Thie treatment is usually correction of the unde lying metabolic abnormality.

The spike-wave discharges accompanyifg spike-wave stupor are medium to hig amplitude, generalised, predominanty fronto-midline, synchronous complexes (surface negative spike followed by surfage negative slow wave). The morphology may be less regular than the spike-wave of typical absence seizures, and polyspikes may be admixed. ${ }^{5}$ These repetitive spike-wave coff plexes fire rhythmically at a frequency of $2 \pm 0$ $6 \mathrm{~Hz}$ (usually $2 \cdot 5-3 \cdot 5 \mathrm{~Hz}$ ). ${ }^{67}$ The complexes . may be continuous, or they may occur in repetitive bursts alternating with normal 6 . near-normal background activity. A strong external stimulus may temporarily $\mathbb{H}$ malise the EEG. ${ }^{5}$

Spike-wave stupor (absence status) \$ \& epileptic phenomenon clinically manifegitig as a confused state lasting minutes to days. In most instances, it occurs as part of a pisexisting seizure disorder, usually absenoe sê.zures. $^{56}$ The treatment is usually by anf epileptic medications. ${ }^{9}$

This case report illustrates how an IfEG finding (triphasic waves) combined wh appropriate laboratory and radiologic confirmation can differentiate the diagnosis of portal-systemic encephalopathy fro other causes of episodic obtundation, including spike-wave stupor. Yamamcto and Hosokawa correctly point out thet spike-wave stupor has occasionally been reported in patients without a previous seizure history. Rarely, the condition may be associated with acquired neurological disease (head injury, encephalitis, cepebrovascular disease) ${ }^{6}$ or predisposing toxitmetabolic disturbances. ${ }^{10}$ The saîfe associations occur with typical absence sejzures. $^{9}$

The EEG distinction between triphasic waves and spike-wave discharges makes EEG useful in ensuring that metabolic disorders and epileptic spike-wave stupor fe each diagnosed and treated appropriatel

PHILIP H SHERID SUSUMU SATO

National Institute of Neurologiça 
and Communicative Disorders and Stroke National Institutes of Health, Bethesda, Maryland USA

Postal address: Dr Philip H Sheridan, Federal Building, Room 114, NINCDS, Bethesda, MD, 20205 USA

\section{References}

${ }^{1}$ Yamamoto M, Hosokawa K. Triphasic spikewave stupor in portal-systemic encephalopathy: a case report. J Neurol Neurosurg Psychiatry 1985:48:386-7.

${ }^{2}$ Bickford RG, Butt HR. Hepatic coma: the electroencephalographic pattern. J Clin Invest 1955:34:790-9.

${ }^{3}$ Spehlmann R. EEG Primer. Amsterdam: Elsevier/North Holland Biomedical Press, 1981:327.

${ }^{4}$ Saunders MG, Westmoreland BF. The EEG in evaluation of disorders affecting the brain diffusely. In: Klass DW, Daly DD, eds. Current Practice of Clinical Electroencephalography. New York: Raven Press, 1979:352-7.

${ }^{5}$ Niedermeyer E, Khalifeh R. Petit mal status ("spike-wave stupor"). Epilepsia 1965: 6:250-62.

${ }^{6}$ Hosokawa K, Booker HE, Okumura N, et al. Spike-wave stupor. Folia Psychiatr Neurol Jpn 1970:24:37-47.

${ }^{7}$ Kellaway P, Chao D. Prolonged status epilepticus in petit mal. Electroencephalogr Clin Neurophysiol 1955:7:145.

${ }^{8}$ Lennox WG. The petit mal epilepsies: their treatment with Tridione. JAMA 1945: 129: 1069-74.

${ }^{9}$ Sato S. Generalized seizures: absence. In: Dreifuss FE, ed. Pediatric Epileptology. Boston: John Wright-PSG, 1983:65-91.

${ }^{10}$ Feldman RG. Management of underlying causes and precipitating factors of epilepsy. In: Brown TR, Feldman RG, eds. Epilepsy: Diagnosis and Management. Boston: Little, Brown and Company, 1983:129-38.

\section{Yamamoto et al reply:}

We also believe that episodic behaviour disorders must be differentiated from epileptic disorders and metabolic encephalopathy as Drs Sheridan and Sato note. They concur that spike-wave stupor is rarely found in non-epileptic disorders. We use this term as a state diagnosis or a state description, not as a clinical diagnosis. In portal-systemic encephalopathy, Poser $^{1}$ described the triphasic wave with epileptic nature. In our case, to our regret, the EEG is somewhat ambiguous, but it has bilateral synchronous spike component of epileptic nature. Hosokawa et $a l^{2}$ previously showed that diazepam inhibits the spike-wave discharge in portalsystemic encephalopathy. We believe that spike-wave stupor in portal-systemic encephalopathy could be considered to be due to secondary epileptogenesis caused by metabolic disturbances in that case.

From this standpoint, spike-wave stupor in portal-systemic encephalopathy is not contrasted with epileptic spike-wave stupor.

\section{References}

${ }^{1}$ Poser CM. Electroencephalographic changes and hyperammonemia. Electroencephalogr Clin Neurophysiol 1958:10:51-62.

${ }^{2}$ Hosokawa K, Kogıh T, Otsuki S, et al. A case of the hepatic encephalopathy with unusual episodes of the triphasic spike-wave stupor. Clin Neurol 1977:17:391-7.

\section{Book reviews}

Vestibular and Visual Control on Posture and Locomotor Equilibrium. (7th International Symposium of the International Society of Posturography, Houston). Edited by M Igarashi and FO Black. (Pp $366 \$ 80.50$.) Basel: Karger, 1985.

This book records the proceedings of the 7 th International Symposium of the International Society of Posturography. It contains papers which are either overviews of the state of the art in particular aspects of posturography or accounts of specific experiments. Various sub-sections of the book deal with models of postural control, quantitative techniques, interaction with visual and vestibular systems, the aerospace environment, neurophysiology and developmental and pathological studies.

With the exception of the short section on neuro-physiology, all the papers take what might be termed as a "behavioural" or "human engineering" approach to the study of posture and are largely concerned with studies on human subjects.

As is to be expected with the proceedings of any conference, the quality of individual contributions is variable. However, a substantial number of the $\mathrm{p}^{\text {n }}$ ers are of high quality. The book has the appeal, derived from the multi-disciplinary nature of posturography, that it contains something of interest for almost everybody concerned with behavioural studies of posture.

MA GRESTY
Neurosurgical Operations. By G Merren and W-E Goldhahn. (Pp 556; DM 120.00.) Heidelberg: Springer-Verlag, 1985.

This is the second edition of a standard German language neurosurgical text written by the late Professor Merren and re-edited by Professor Goldhahn, and now translated into English. The book consists of various sections including general neurosurgical techniques, vascular neurosurgery, spinal surgery, pain and stereotaxy. In each section a page is devoted to a description of the operation and the opposite page to one or two detailed sketches illustrating the text. The description of the operations are reasonably clear, and the drawings are more helpful than they first appear; the quality of the reproduction and printing is not high.

The techniques described are orthodox neurosurgery as practised in a major East German centre. The neurosurgeon in training would find it a reasonable basic guide but would expect more detailed correlation. There is no mention of modern equipment such as the CUSA sucker and laser surgery. It is an interesting book but it also has important competitors, whose price is similar, and whose multiple authorship confers the benefit of specialists writing about their particular field of interest.

MICHAEL POWELL

Scientific Basis of Clinical Neurology. Edited by $M$ Swash and C Kennard. (Pp 805; £60.00.) Edinburgh: Churchill Livingstone, 1985.

For many physicians the fascination of clinical neurology is the pleasure of deductive analysis based on careful observation in the light of applied anatomy and physiology. As one's experience matures one must inevitably become more removed from the scientific background which nurtured it. Furthermore, experience shows us that some of the simplistic models which we inherited are in themselves insufficient. In The Scientific Basis of Neurology Swash and Kennard have assembled a series of review articles, not only on the most immediate and glamorous spheres of neurological research, but also defining the current concepts of the most basic and essential aspects of neurological function. There are 67 distinguished contributors, literally extending from A (Appenzeller on the Autonomic Nervous System) to Z (Zeki on Looking and Seeing). The individual reviews are on average some 20 pages in length and any one can be digested at a single sitting. Each chapter is supplemented 